\title{
The specification and testing of a Horizontal Axis Tidal Turbine Rotor Monitoring approach.
}

\author{
Matthew Allmark ${ }^{1}$, Paul Prickett ${ }^{2}$, Roger Grosvenor ${ }^{3}$ and Carwyn Frost ${ }^{4}$ \\ 1,2,3 School of Engineering, Cardiff University, The Parade, Cardiff, CF24 3AA \\ allmarkmj1@cf.ac.uk
}

${ }^{4}$ Queen's University, School of Natural and Built Environment, David Keir Building, Stranmillis Road, Belfast, BT9 5AG
C.Frost@qub.ac.uk

\begin{abstract}
The sustainable deployment of Horizontal Axis Tidal Turbines will require effective management and maintenance functions. In part, these can be supported by the engineering of suitable condition monitoring systems. The development of such a system is inevitably challenging, particularly given the present limited level of operational data associated with installed turbines during fault onset. To mitigate this limitation, a computational fluid dynamics model is used to simulate the operational response of a turbine under a known set of fault conditions. Turbine rotor imbalance faults were simulated by the introduction of increasing levels of pitch angle offset for a single turbine blade. The effects of these fault cases upon cyclic variations in the torque developed by the turbine rotor were then used to aid creation of a condition monitoring approach. A parametric tidal turbine rotor model was developed based on the outputs of the computational fluid dynamics models. The model was used to facilitate testing of the condition monitoring approach under a variety of more realistic conditions. The condition monitoring approach showed good performance in fault detection and diagnosis for simulations relating to turbulence intensities of up to $2 \%$. Finally, the condition monitoring approach was applied to simulations of $10 \%$ turbulence intensity. Under the $10 \%$ turbulence intensity case the rotor monitoring approach was successfully demonstrated in its use for fault detection. The paper closes with discussion of the effectiveness of using computational fluid dynamics simulations extended by parametric models to develop condition monitoring systems for horizontal axis tidal turbine applications.
\end{abstract}

\footnotetext{
Matthew Allmark et al. This is an open-access article distributed under the terms of the Creative Commons Attribution 3.0 United States License, which permits unrestricted use, distribution, and reproduction in any medium, provided the original author and source are credited.
}

\section{INTRODUCTION}

Energy extraction from the ocean's tides has gained widespread acceptance as a potential contributor to the UK energy mix (Department of Energy \& Climate Change, 2013). The driving factor behind the uptake in tidal energy extraction has been the realisation of finite global resources and environmental impacts of burning fossil fuels (Zhang \& Zeng, 2013). In order for the Horizontal Axis Tidal Turbine (HATT) devices to generate energy at a competitive levelized cost of energy (LCOE), effective strategies for reducing the burden of operation and maintenance $(\mathrm{O} \& \mathrm{M})$ costs are needed. This has led to the research and development of condition monitoring (CM) systems to facilitate condition-based maintenance operations. These aim to reduce $\mathrm{O} \& \mathrm{M}$ costs and prevent catastrophic failure and premature replacement of turbine components. In the context of the more mature wind energy sector $\mathrm{O} \& \mathrm{M}$ costs associated with wind turbine installations have been quoted as accounting for up $25 \%$ of the asset cost (Godwin \& Matthews, 2013).The approach of utilising CM to inform Condition Based Maintenance (CBM) operations is likely to be appropriate for the HATT energy sector where $O \& M$ costs are likely to be exacerbated by the harsh marine environments within which HATTs are deployed. The paper presents the development of a HATT rotor monitoring approach developed using CFD data. This approach is heralded, relative to the data driven approaches developed by (Leahy et al., 2018) and (Hasegawa, Ogata, Murakawa, Kobayashi, \& Ogawa, 2017) for Wind Turbines (WTs), as limited Supervisory Control and Data Acquisition (SCADA) data is currently available for TST deployments.

The CM techniques presented in this paper were developed in two stages: firstly, a series HATT rotor imbalance measures were developed. These CM metrics are based on established Computational Fluid Dynamics (CFD) HATT models. These models were developed to harbour varying degrees of erroneous pitch angle settings for a single turbine 
blade thus simulating differing levels of rotor imbalance fault. The frequency content of the torque developed by the HATT rotor, under the varying rotor imbalance conditions, was inspected to guide development of a set of CM metrics. The aim of this approach was to develop a set of CM metrics based on resolved CFD models under near-steady conditions. In this way, the underlying effects of the fault cases were observed and exploited during CM metric development. Furthermore, these effects observed in CFD simulations by association are then based on validated models. The inference applied was that, using validated models and studying the underlying blade loading mechanisms associated with the fault cases considered, an intelligent CM approach could be developed.

Secondly, the research then considered the development of a parametric rotor torque model. This model is used to generate time-series of the developed HATT rotor torque which are representative of general and stochastic turbine operations i.e. turbine operation under varying levels of turbulence intensity (TI) in the on-coming flow. Modelling such scenarios via CFD is computationally expensive and time consuming; especially, as a minimum number of datasets are generally required to afford statistical significance to the CM study. As such, the approach utilises a novel parametric rotor model which was engineered in order to provide numerous datasets to which the developed algorithms could be applied. This approach is then deployed to refine and test the HATT rotor fault monitoring process that was developed based on the aforementioned frequency domain characteristics observed in the CFD data. Essentially, the parametric model was created to extend the useful data provided by CFD simulations. The effectiveness and the practicality of implementing such algorithms to support future CM system developments are then considered.

The paper presents a brief review of the literature relating specifically to CM systems within the context of HATT. It then considers the nature and formulation of the CFD data utilised. The development of the rotor imbalance monitoring processes are then outlined. The parametric rotor torque model is then presented and utilised to test the effectiveness of the CM approaches under varying levels of TI. Next, the initial simulation results, for turbulence intensities varying from $0.5 \%$ to $2 \%$ are presented along with the condition monitoring results. An extended results section details the application of the monitoring approaches to a data set developed utilising a more realistic fluid velocity simulation approach based on methods reported in (Val, Chernin, \& Yurchenko, 2014). In this section, the CM approaches are applied to data relating to HATT operation within flows of $3.086 \mathrm{~ms}^{-1}$ with TI of $10 \%$. The paper presents discussion of the effectiveness of the overall strategy for developing $\mathrm{CM}$ approaches in light of minimal operational data. Conclusions based on the work presented are drawn; lastly and for brevity, the details of the method for generating stochastic fluid velocity time-series is included in Appendix I.

\section{REVIEW OF TST CONDITION MONITORING}

The improvement and assurance of the reliability of HATTs and their sub-assemblies must be considered to be a major factor in the realisation of a well-functioning tidal stream energy industry. HATTs are to face operation in the harsh marine environment and are to be exposed to cyclic and extreme loading. Cyclic loading is enforced on the tidal stream device by both the presence of turbulence in the fluid field and due to semi-diurnal tidal cycles which dominate the UK tidal resource. Mixed tidal cycles are found in many other areas where tidal stream energy extraction is feasible. It has been argued that in order to achieve a competitive LCOE component and turbine availability should be above $75 \%$ (Magagna et al., 2014). In moving toward a higher technology readiness level (TRL) and to underpin the significant levels of investment required it has also been stated that the reliability of HATTs and their components must be demonstrated (Wolfram, 2006); (Weller, Thies, Gordelier, \& Johanning, 2015).

Caselitz et al (Caselitz \& Giebhardt, 2005) presented one of the earliest papers aimed specifically at the condition monitoring of HATTs. The paper aimed to apply and adapt knowledge acquired in the condition monitoring of both on-shore and off-shore WTs to the task of CM of HATTs.They propose a system containing many of the elements included in WT monitoring systems, including accelerometers for gearbox and baring vibration monitoring and fibre bragg gratings for blade and structural load monitoring. The system is supported by environmental measurements, specifically by measurements of the fluid velocity upstream of the turbine rotor. Sloan et al (Sloan, Khoshgoftaar, Beaujean, \& Driscoll, 2009) presented considerations of many of the reliability issues faced by HATTs and the associated monitoring hardware. Mjit et al (Mjit, Beaujean, \& Vendittis, 2010) conducted work considering order analysis of vibrational data as a means of fault detection, the work was first conducted on a commercial fan and later extended to monitoring of a small boat propeller. The work also discussed elements of the data storage and capture processes. The order analysis methods applied were successful in identification of imbalance and misalignment. The research undertaken and presented by Mjit et al was extended in 2011 (Mjit Mustapha, Beaujean, \& Vendittis, 2011) to incorporate a more fully developed Smart Vibration Monitoring System (SVMS) which handled much of the data capture storage and processing autonomously. The system included many of the techniques performed off-line by vibration monitoring specialists including advanced signal processing of vibration data. Specifically, the software processed raw vibrational data via Power Spectral Density, Fractional Octave, Cepstrum, Hilbert Envelope, Wavelet Transform and overall vibrational statistical characteristics. The process was devel- 
oped using LabVIEW and tested using a drive train test rig setup to harbour fault conditions by attaching weights to the drive train. Changes to the performance metrics calculated via the advanced signal processing operations deployed were successfully tracked for three differing levels of fault severity and for two rotational velocities.

Waters et al (Waters, Beaujean, \& Vendittis, 2013) presented research considering the detection, localisation and identification of bearing faults in TST applications. The paper presents studies within which models for bearing loading under bearing race cracking were developed. The models were then used to guide the development signal processing methods which were then applied to vibration signals acquired from two accelerometers mounted on dynamometer test beds. In order to detect bearing damage the power spectral density of the measured accelerometer signals was utilised as well as the coherency between them. A fault condition was said to exist if the power observed at frequencies of interest (found via impact test) exceeded a given threshold. The fault was then localised to a given bearing by comparison of the power spectral density measurements at the frequencies of interest from the two accelerometer measurements. Envelope tracking was then used to identify the fault type (inner vs outer race fracture) by considering the timing of the observed impacts yielding the observed increases in spectral power at the frequencies identified.

\section{CFD Simulations}

Cardiff marine energy research group (CMERG) has extensive expertise in producing and validating CFD models (MasonJones et al., 2012), (Tatum, Frost, et al., 2016),(Tatum, Allmark, et al., 2016). Recent advances in the modelling approach utilized within the group led to the production of transient CFD models (Frost, Morris, Mason-Jones, O’Doherty, \& O’Doherty, 2015). Such models offer an opportunity to study turbine loading throughout a single turbine rotation under differing operating conditions. Along with the ability to study turbine loading transients, these models can be set-up to simulate rotor imbalance defects, which could be compared to the optimum condition for turbine rotor monitoring studies.

The CFD model used in this work adopted a $5 \mathrm{~m}$ radius rotor equipped with three adapted Wortman FX-63 -137 blades, a schematic of the rotor set-up is shown in Figure 1. The model was configured with constant fluid velocity at the model inlet, both in time and spatially (plug-flow). The fluid domain for the model was $150 \mathrm{~m}$ by $50 \mathrm{~m}$ by $50 \mathrm{~m}$ and utilised a moving reference frame at the turbine rotor. The mesh had approximately 6 million elements and the model utilised a shear stress transport viscous model. In order to conduct an initial study into TST rotor monitoring this knowledge base was utilised to provide CFD model results for differing rotor conditions. The inlet fluid velocity was set to $3.086 \mathrm{~ms}^{-1}$. The turbine rotational velocity was set to $2.1 \mathrm{rads}^{-1}$ resulting in

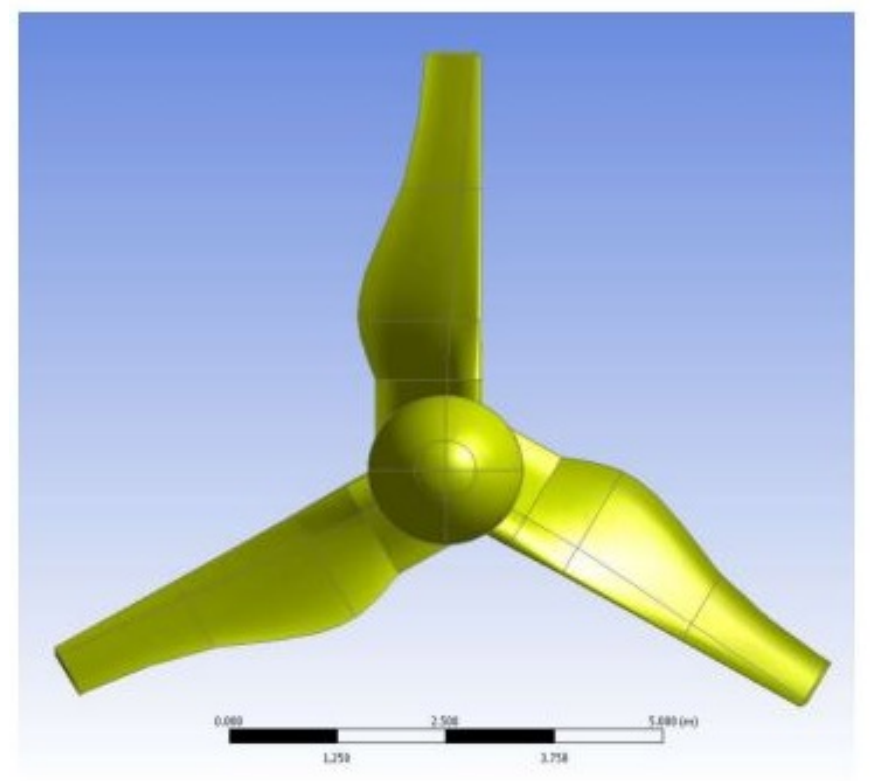

Figure 1. Schematic of the three bladed turbine rotor simulated.

a tip speed ration $\lambda$ value of 3.61 for each of the simulations. This corresponds to operation at the peak power coefficient for the rotor used. The previous research undertaken within the group provided a high level of confidence that the data sets produced would be representative of the expected turbine characteristics. This was seen as being important going forward as the intention was to produce robust $\mathrm{CM}$ algorithms that could be widely applied.

The previous research had established that the optimum blade pitch angle, balancing power extraction and thrust loading, was at $6^{\circ}$ to the rotor plane (Mason-Jones, 2010). Figure 2 shows the data output from the CFD models for the optimum and blade 1 pitch angle offset by $+6^{\circ}$ rotor setting

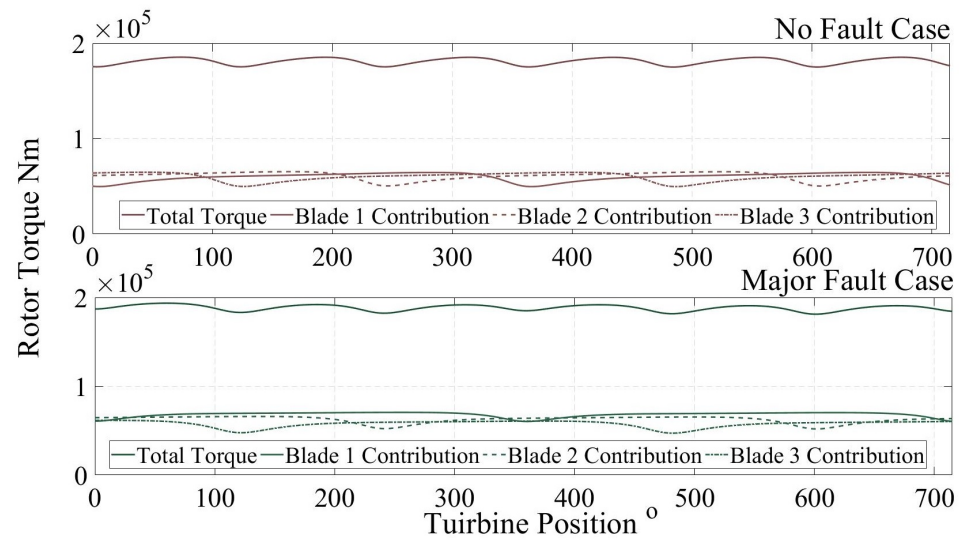

Figure 2. Plots showing the torque developed by the TST rotor as calculated via CFD. 
Table 1. Table showing the fault cases simulated via both CFD and random parametric model simulations for development of CM apporaches.

\begin{tabular}{|c|c|}
\hline No Fault Case & Pitch Angle: $6^{\circ}$ \\
\hline Sensitivity Case & Pitch Angle: $6^{o}+0.5^{\circ}$ \\
\hline Minor Fault & Pitch Angle: $6^{o}+3^{\circ}$ \\
\hline Major Fault & Pitch Angle: $6^{\circ}+6^{\circ}$ \\
\hline
\end{tabular}

cases. The figure highlights both the cyclic nature of the drive shaft torque imposed by the rotor and the form of the results outputted from the CFD modelling exercise. For convenience the data was output in the form of the overall torque imposed on the turbine drive shaft by the HATT rotor and the contribution by each blade to the developed turbine drive shaft torque. This was exploited in the initial steady state simulations where the overall rotor torque contribution was considered for condition monitoring process development. This was further utilised to construct a parametric rotor torque model. Along with the optimal blade pitch set-up three differing blade 1 pitch offset cases were simulated to give a range of fault severities. Table 1 shows the cases simulated. The four cases include a Non Fault case as well as three fault cases of increasing imbalance severity. In the next section 4 , the data will be discussed in terms of its use in development of the CM metrics proposed.

\section{ROTOR CM APPROACH SPECIFICATION}

The approach to the development of the CM algorithm was based on study of the output of the CFD models detailed in Table 1. To this end, the spectra of each of the developed rotor torque time-series for each fault case, were studied. This was done to view changes in torsional oscillations due to the rotor imbalance conditions which could be exploited for fault detection and diagnosis. Figure 3 shows the developed rotor torque spectrum in the turbine displacement domain - accordingly the $\mathrm{X}$-axis is plotted with units $1 / \theta^{\circ}$, denoted here as $H z_{\theta}$. The figure shows the spectra for each of the rotor cases detailed in 1 . The fluid velocity and rotational velocities are as detailed above.

The peak amplitudes observed in each of the torque spectra were found to exist at three times per turbine rotation $\left(0.00833 H z_{\theta}\right.$ or $\left.3 / 360 H z_{\theta}\right)$ - this has been highlighted in the sub-axis included in Figure 3. Based on previous experience this was attributed to the blade passing the support stanchion or shadowing effect' (Allmark, 2016). Harmonics of this dominant amplitude, at integer multiples of 0.00833 $H z_{\theta}$, were also observed. The amplitudes of these harmonics reduce with increasing harmonic number and are easily observable until the 12th harmonic of the turbine rotational speed. Furthermore, it was observed that the amplitude at the

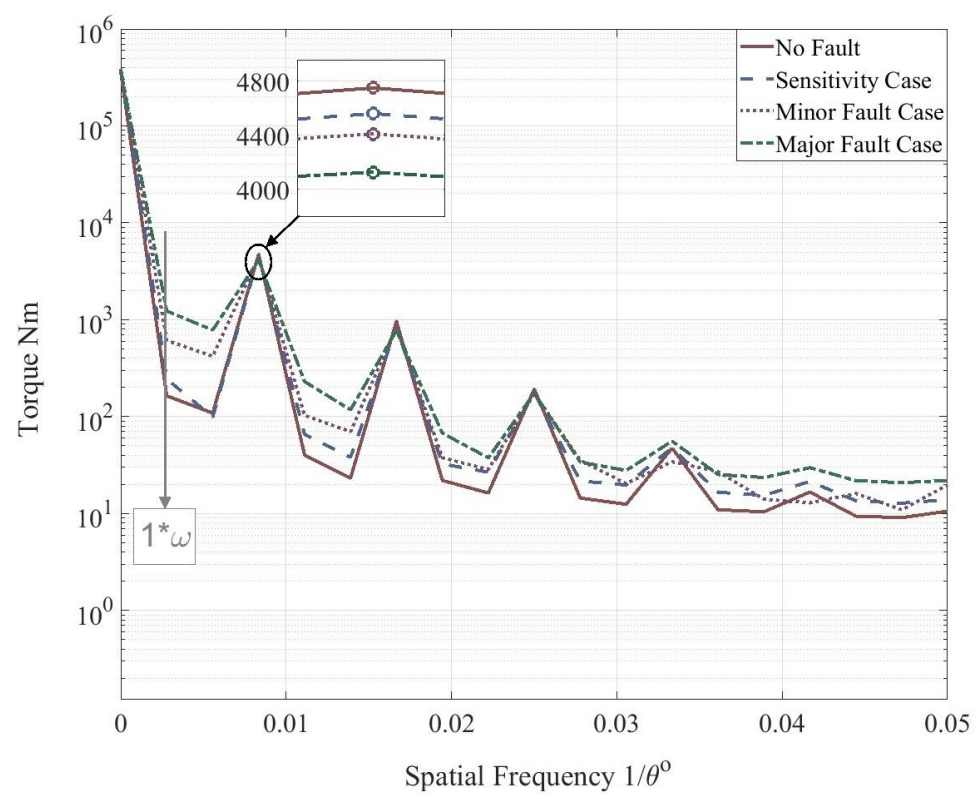

Figure 3. Plots showing the torque developed by the TST rotor as calculated via CFD.

shadowing frequency is reduced with increased rotor imbalance.

Interestingly it was noted that the amplitude at the rotational frequency of the turbine $\left(0.00278 \mathrm{~Hz}_{\theta}\right)$ is increased with increasing levels of rotor imbalance or damage. This was considered to coincide with the increase in variability, and often peak amplitude, of the set of rotational frequency harmonics which were not multiples of three, i.e. integer multiples of the blade passing amplitude found at $0.0833 \mathrm{~Hz} z_{\theta}$.

Analysis of the model outputs shown in Figure 3 allowed the authors to propose a number of condition monitoring metrics. These were based, in particular, on the amplitude shifts outlined above for differing levels of rotor damage. Using $A_{i \omega}$ to denote amplitudes associated with harmonics of the turbine rotational velocity, where the subscript $i$ refers to the harmonic number, the monitoring metrics developed are detailed in Table 2. The application of the monitoring criteria listed in table 2 will be undertaken utilising the discrete-time Fourier transform (DFT) to calculate the required harmonic amplitudes. Data from the stochastic simulations detailed in section 5 will be collected in a number of datasets of 300 second intervals. More detail on data sets structuring will be given in Section 6.

\section{Turbine Rotor Simulation}

\subsection{Fluid Velocity Simulation - Initial Approach}

To develop and test the validity of the proposed CM metrics a simple resource simulation method was applied and coupled with the parametric model outlined in the next Section. The 
Table 2. Table showing the four CM metric developed based on the spectra observed within the CFD data for differing fault cases.

\begin{tabular}{c}
$1 \cdot \omega$ Energy \\
$\left|A_{1 \cdot \omega}\right|^{2}$ \\
$3 \cdot \omega$ Energy \\
$\left|A_{3 \cdot \omega}\right|^{2}$ \\
\hline $1 \cdot \omega$ to $3 \cdot \omega$ Energy Ratio \\
$20 \log \left(\frac{\left|A_{1 \cdot \omega}\right|^{2}}{\left|A_{3 \cdot \omega}\right|^{2}}\right)$ \\
\hline $\begin{array}{c}\text { Rotational harmonic energy } \\
\text { excluding } 3 \cdot \omega \text { harmonic energy } \\
6 \\
\sum_{i=1}\left|H(i) A_{H(i) \cdot \omega}\right|^{2} \\
H=[1,2,4,5,7,8] \\
w h e r e\end{array}$ \\
\hline
\end{tabular}

model utilised was of the following form:

$$
U_{x}(t)=\bar{U}_{x}+u_{x}^{\prime}
$$

where $U_{x}(t)$ is the fluid velocity at time $t$ decomposed into a stationary mean fluid velocity $\bar{U}_{x}$ and a fluctuating component, $u_{x}^{\prime}$ which is time varying with the $\mathrm{x}$ direction perpendicular to the turbine rotor plane. The fluctuating component for this study was simulated via a normally distributed random variable with zero average and with the standard deviation required for the specific TI. TI here is defined as:

$$
T I=\frac{\sigma_{u}}{\bar{U}_{x}}
$$

where, $\sigma_{u}$ is the standard deviation of the fluctuation fluid velocity component, $u_{x}^{\prime}$.

\subsection{Parametric Rotor Model}

In order to construct a parametric rotor model the effects of rotor transients, as seen in the transient CFD data, were combined with the expected mean rotor torque for a given operating condition. The mean rotor torque developed by a turbine is given by:

$$
T_{c_{\tau}}(t)=c_{\tau} \cdot \frac{1}{2} \cdot \rho \cdot A \cdot r \cdot U_{x}^{2}
$$

where, $c_{\tau}$ is the non-dimensional torque constant, $\rho$ is the fluid density, $A$ is the swept area of the turbine, $r$ is the turbine radius and $U_{x}$ is the on coming fluid velocity. The $c_{\tau}$ term can be found using the non-dimensional parameters curves for the given turbine rotor, previous work has detailed both CFD and experimental validation of the non-dimensional curves for the rotor under investigation (Mason-Jones et al., 2012).

A flow chart representing the parametric model, utilising the mean torque calculated via (7), is provided in Figure 4. This identifies the inputs into the process from simulated fluid velocity data and the turbine parameters extracted from the CFD data detailed in Section 3. For a given time step the fluid velocity, as well as the turbine position and rotational velocity (which is fixed for these simulations) are in-putted. These quantities along with the characteristic curves and parameter set for the given TST rotor are then used to calculate the expected torque developed by the turbine rotor for the given flow speed and condition. The output of the model is then a simulated rotor torque time series.

The periodic nature of the torque fluctuations under various rotor conditions have accordingly been captured via a parametric model of the form of three Fourier series, one for each blade. The remainder of this section outlines the structure and parameter set of the parametric model. The parameter set and model structure were developed by considering the amplitude and phase spectra for each individual blade contribution to the torque developed by the turbine rotor. Figure 5 shows the amplitude spectra for each blade. Cases shown are Optimum or No Fault and the Major Fault case where blade 1 has been set to $+6^{o}$ from the optimum pitch setting. The fluid velocity and rotational velocities are as detailed above.

The frequency index for the spectra, in the form used in Equation (8) is shown above the figure and the harmonic indexes are identified. It can be observed that blade offsetting distorts the harmonic content of the frequency domain representation from the optimum case. Each spectrum also exhibits an exponentially decaying tendency with differing peak values and decay rates. This observable exponential decay over multiple harmonics of the turbine rotation was utilised in the parametric model as can be seen in Equation (8). The terms in Equation (8) have been included in the flow chart of the simulation process depicted in Figure 4 to further indicate the structure of the parametric turbine rotor model. The parametric model utilised a Fourier series of the form: 


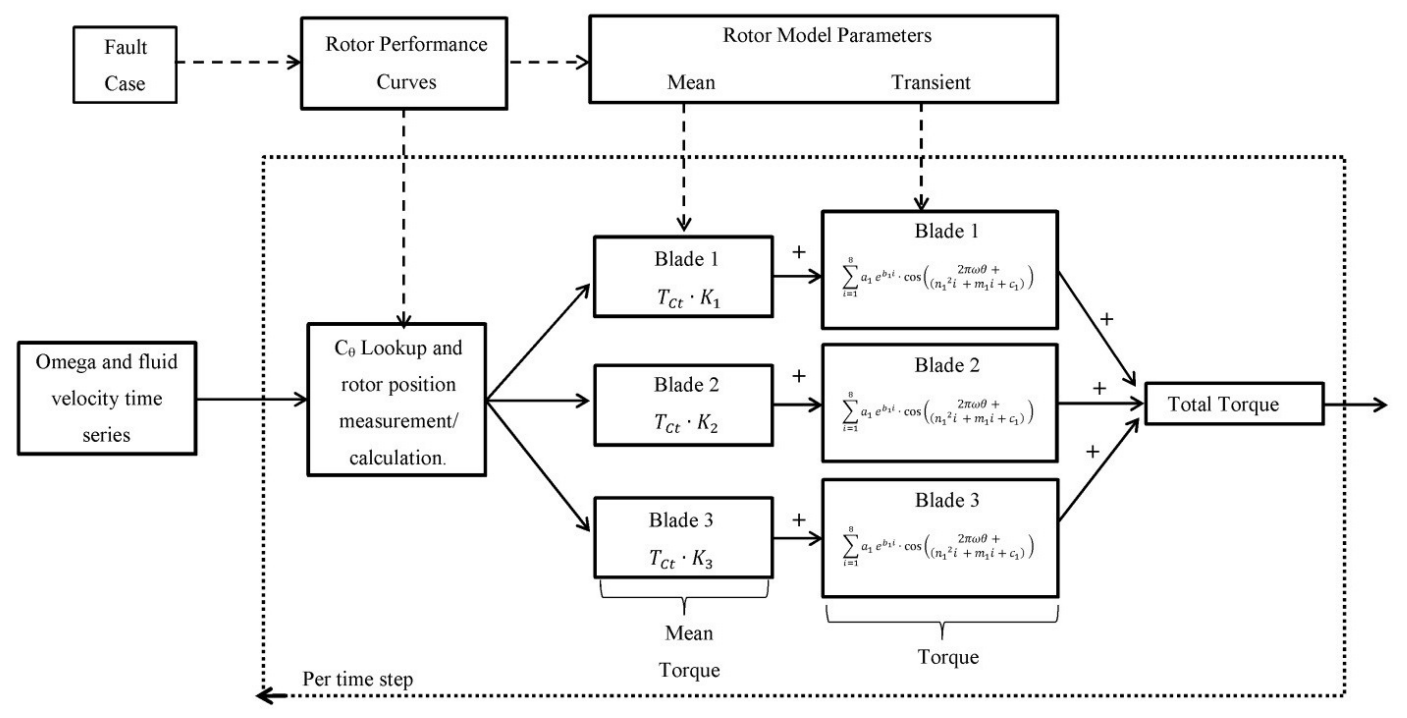

Figure 4. A schematic showing the structure of the Parametric model used to generate stochastic simulations of the outlined TST.

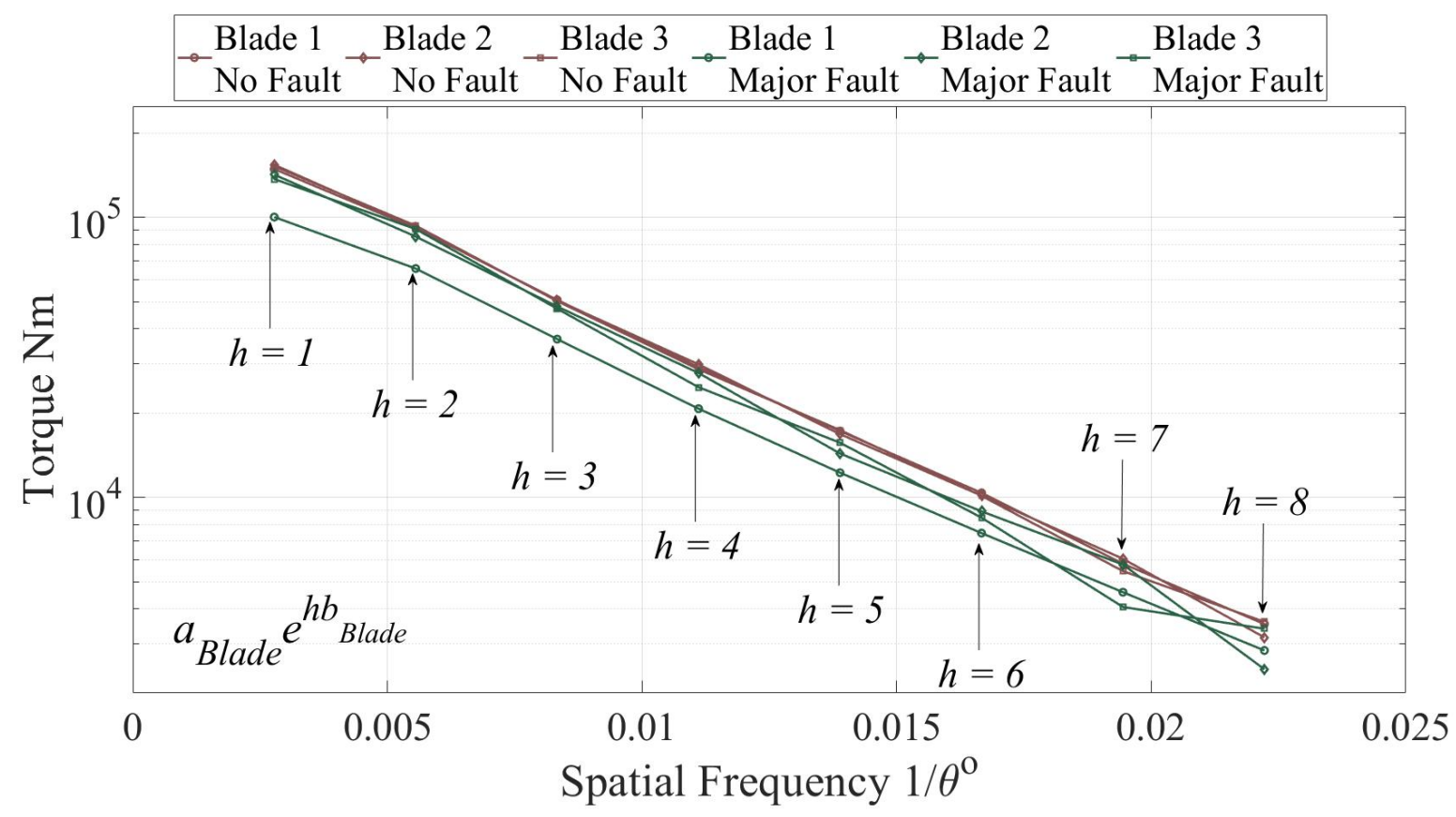

Figure 5. Plots showing the torque amplitude spectra developed per blade by the TST rotor as calculated via CFD. 


$$
\begin{array}{r}
T_{\text {rotor }}=T_{c_{\tau}} \cdot\left(k_{1}+\sum_{h=1}^{8} a_{1} e^{b_{1} h} \cdot \cos (2 \pi \omega \theta+\right. \\
\left.\left.\left.+n_{1}^{2} h+m_{1} h+c_{1}\right)\right)\right) \\
+T_{c_{\tau}} \cdot\left(k_{2}+\sum_{h=1}^{8} a_{1} e^{b_{2} h} \cdot \cos (2 \pi \omega \theta+\right. \\
\left.+T_{c_{\tau}} \cdot\left(k_{2}^{2} h+\sum_{h=1}^{8} a_{1} e^{b_{3} h} \cdot \cos \left(2 \pi \omega \theta+c_{2}\right)\right)\right) \\
\left.\left.\left(n_{3}^{2} h+m_{3} h+c_{3}\right)\right)\right)
\end{array}
$$

It can be seen in (8) how the relationship between the observed phase angles at each harmonic for differing rotor conditions was captured via the parametrisation of the quadratic equation with a set of three parameters $\mathrm{m}, \mathrm{n}$ and $\mathrm{c}$ for each rotor condition. It was necessary to apply a sequence of phase angle shifts at each harmonic to create consistency between the phase spectra observed for blades 1,2 and 3. This phase shifting process was applied to the phase spectra observed for each rotor case. The results of the phase shifting process applied to the optimum and $+6^{\circ}$ offset cases are shown in Figure 7 , along with the parametric form utilised to model the phase angles observed for each harmonic number for differing blades and rotor conditions. The analysis of this and similar results for the other offset cases confirmed the appropriateness of the choice of $2^{\text {nd }}$ order polynomial form utilised within the parametric model.

The focus of this initial investigation was, by necessity, on TST operation at close to peak power conditions, rather than across the entire power curve. Accordingly, parameters were determined for a tip speed ratio of 3.6. Although it was likely that, as a result of the fluid velocity fluctuations, a wider TSR range would be observed the constant parameter assumption was deemed acceptable for the current baseline study. This assumption allowed the parameters in the model to be held constant relative to the TSR and parameterisation to be undertaken utilising data from a single operating condition (as was the format of the CFD data). Then referring to (8) the parameter set was as follows:

$$
\begin{aligned}
& k \text { is the blade torque contribution for a given TSR } \\
& a \text { is the depth of shadowing effect } \\
& b \text { is the harmonic decay of the shadowing effect } \\
& n \text { is the phase non-linearity } \\
& m \text { is the phase gradient } \\
& c \text { is the phase offset. }
\end{aligned}
$$

The parameter $\mathrm{k}$ gives the relative contribution of each blade to the total drive shaft torque; this in effect sets the DC value of the torque for a given TSR. The parameters $a$ and $b$ give the depth of the shadowing effect and the rate of decay of the eight harmonics for each blade; this in effect defines the magnitude of torque fluctuations due to the aforementioned shadowing effect. Lastly the parameters $\mathrm{m}, \mathrm{n}$ and $\mathrm{c}$ define the phase relationships over the eight harmonics for each blade.

\section{INITIAL RESUlTS}

In order to appraise the effectiveness of the CM metrics as extracted from the rotor torque spectra, a set of TST drive train torque time series simulations were developed. The simulations were generated with a $3.086 \mathrm{~ms}^{-1}$ free-stream flow velocity. Each of these was 200 seconds in length. The time step for the simulations was 0.01 equating to 20,000 samples. In all simulations the turbine rotational velocity was held constant at $2.23 \mathrm{rads}^{-1}$, leading to a $\lambda$ value of 3.6. This setting was providing approximately peak power for the rotor under investigation. For each rotor condition, 50 simulations were undertaken. These were conducted for various levels of TI to further interrogate the ability of the developed CM metrics to extract fault features under the imposed stochastic conditions. Specifically, the datasets were produced with TIs of 0 $\%, 0.5 \%, 1 \%$ and $2 \%$. The levels of TI were selected to coincide with approximately steady-state turbine operation, i.e to constrain the variation in operational $\lambda$. The next sections provide an overview of the simulation results and the application of CM metrics.

\subsection{Simulation Results}

Figure 8 shows the parametric model output for the torque developed by the turbine rotor with $\mathrm{TI}=0 \%$ for both the, 'No Fault' and 'Major Fault' cases. Plotted on the same axis are the CFD data for the same cases, respectively. An average Root Mean Squared Error (RMSE) of approximately 0.0551 $\mathrm{Nm}$ was obtained. Figure 9 shows an example of the simulation results. The figure shows the model output for the optimum rotor case with increasing levels of turbulence. The first case has no turbulent loading and the time series patterns correspond to the reported frequency content for the torque models. The three other cases have increasing turbulences, set at $0.5 \%, 1.0 \%$ and $2.0 \%$ of the mean flow velocity respectively.

\subsection{Condition Monitoring Results}

For each of the 50 simulations at each TI setting and fault case, the condition monitoring metrics were calculated. The CM metrics were calculated utilising the definitions in Table 2 and the spectrum calculated for each simulation. Figure 10 shows the spectra observed for differing fault cases for a set of simulations relating to a TI setting of $2 \%$. The figure highlights the position of the rotational frequency of the tur- 


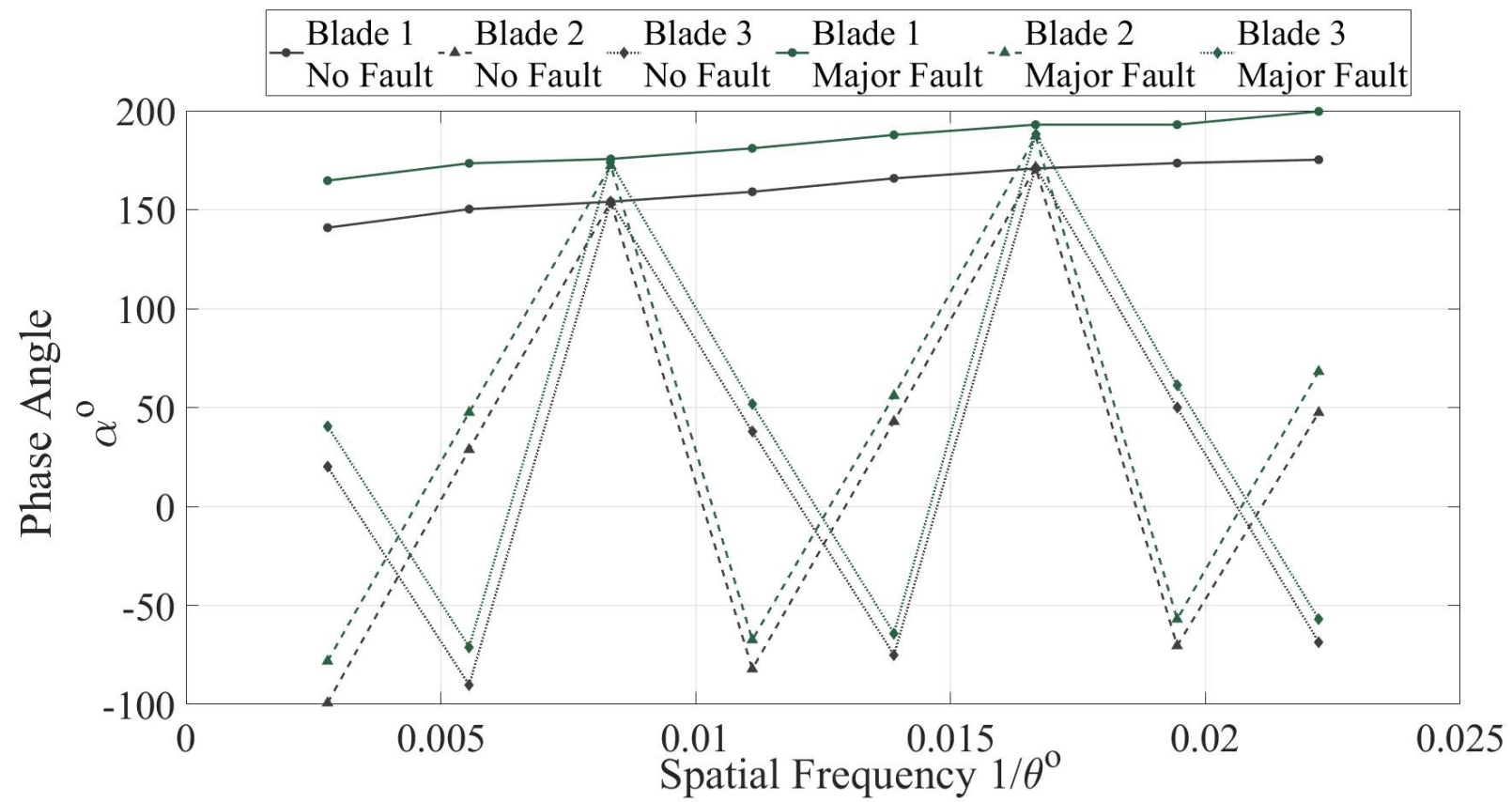

Figure 6. Plots showing the phase spectra for each blade contribution to the torque developed by the turbine rotor prior to unwrapping.

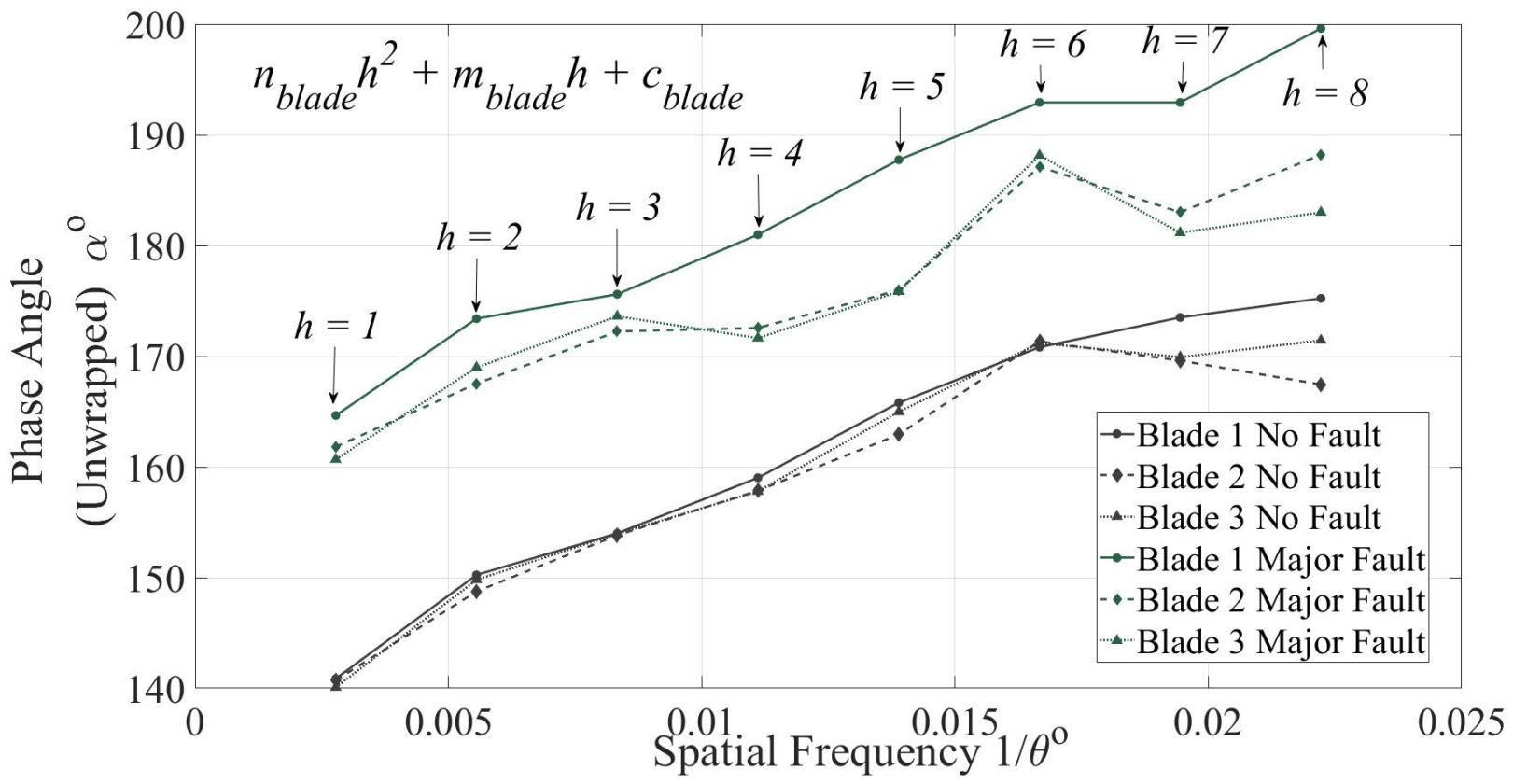

Figure 7. Plots showing the phase spectra for each blade contribution to the torque developed by the turbine rotor after the unwrapping process. 

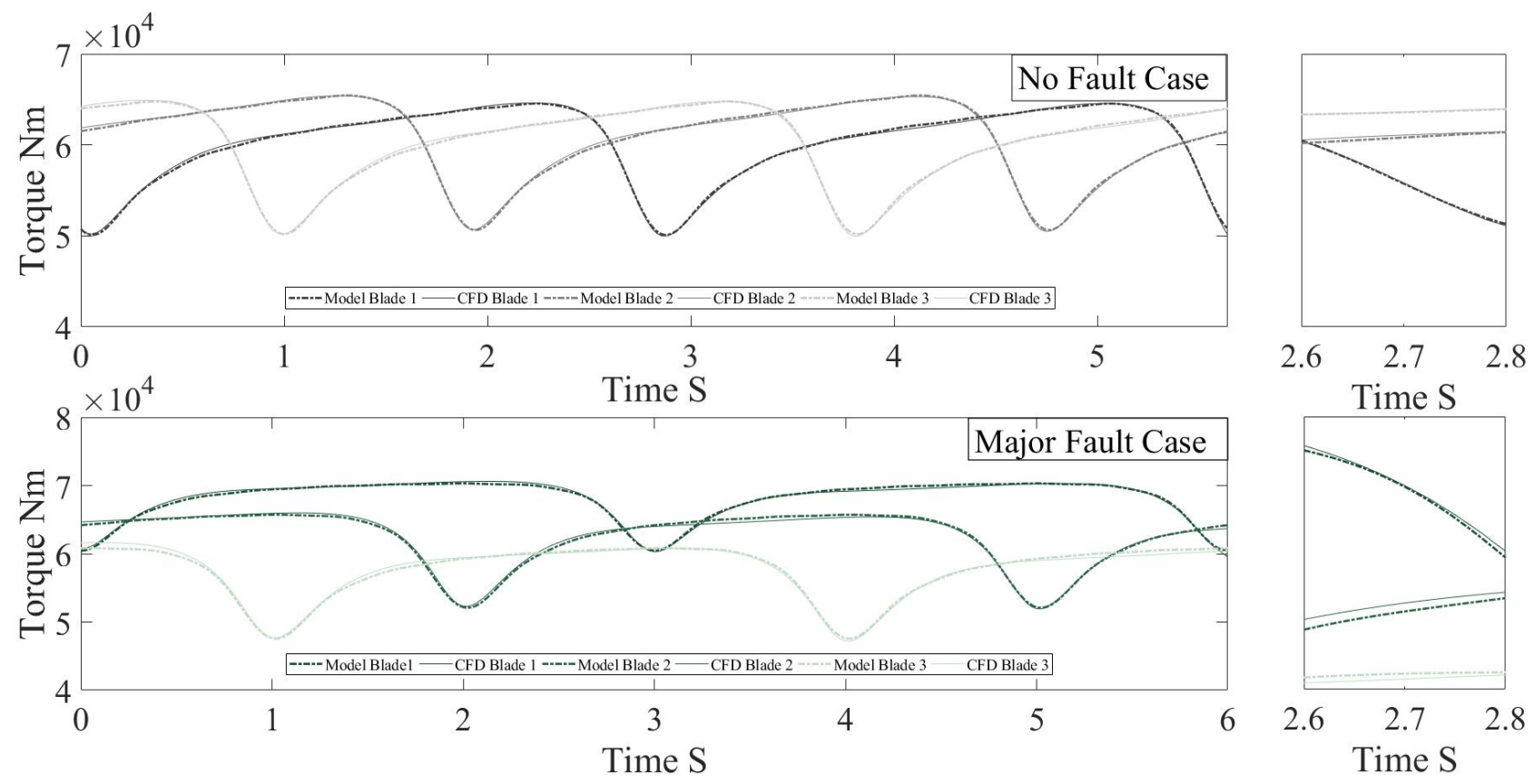

Figure 8. Comparison of the parametric rotor torque model output with the CFD data used to parametrise the model.

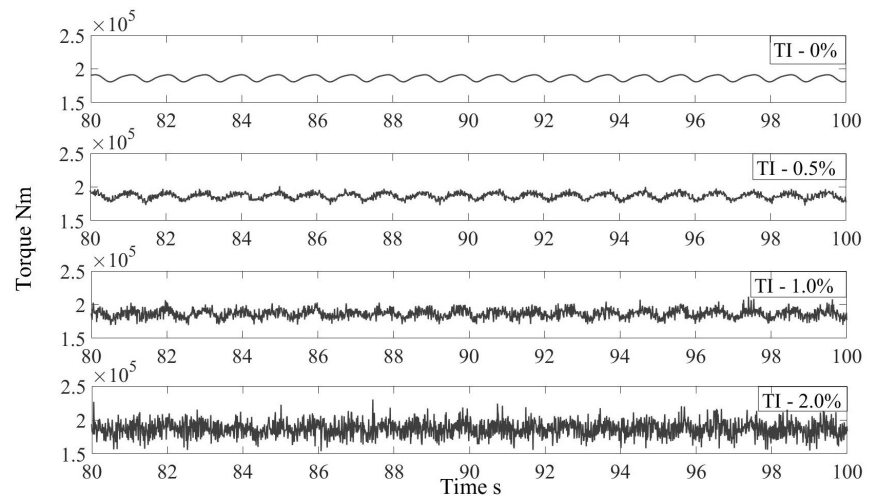

Figure 9. A sample of the simulation results for differing levels of TI in the on-coming flow.

bine; as expected, growth in the amplitude observed at the rotational frequency of the turbine can be seen with increasing fault severity. The blade passing frequency can also been observed as the major amplitude in the spectra; this was expected based the CFD results.

The set of four condition monitoring metrics were then viewed as 4-tuple, 'fault vector'. The metrics associated with each simulation then created a point in the 4-dimensional space associated with the fault vector. To improve the outputs of any machine learning algorithms applied to the CM data each of the metrics were normalised. This was done by subtracting the mean value of the given metric and then dividing the metric by the standard deviation of the observed values of the given metric. Figure 11 shows the normalised CM metrics.
The figure shows the axes associated with the six principle rotations of the 4-D space defined by the aforementioned 4tuple fault vector. It indicates that generally there is clustering of like fault cases, the spread of the clusters for each fault case increases with TI. This suggests the CM metrics could be used to adequately detect and diagnose the fault cases simulated. Lastly, it should be noted, that upon application of the turbulence intensity differing fault cases have little impact on the value CM4.

To illustrate the effectiveness of the CM metrics to distinguish between fault cases, a Naive Bayesian Classifier was trained and used to classify fault conditions given new data. To do this, for each case the 4-tuple fault vectors calculated using the 50 sets of simulation data for each fault case were split in half via random sampling. The randomly selected data sets, consisting of 25 instances of the four monitoring metrics for each fault case, were used to train the classifier. The remaining data sets were used to test the predictions made by the classifier. The prior probability of each of the fault cases where set as equal to yield a, 'neutral' test of the effectiveness of the monitoring approach. The likelihood term, i.e. $P($ Data $\mid$ State $)$ in the standard probability notation, was estimated by fitting normal distributions to the annexed training data for each case. Figure 12 shows the posterior probability of each fault case for the differing input data calculated in the standard fashion - a logarithmic scale is used for the posterior probability axes (y-axis) to show the posterior probabilities observed for the incorrect classification. In all cases a very high posterior probability $(\approx 1)$ was observed for the correct 


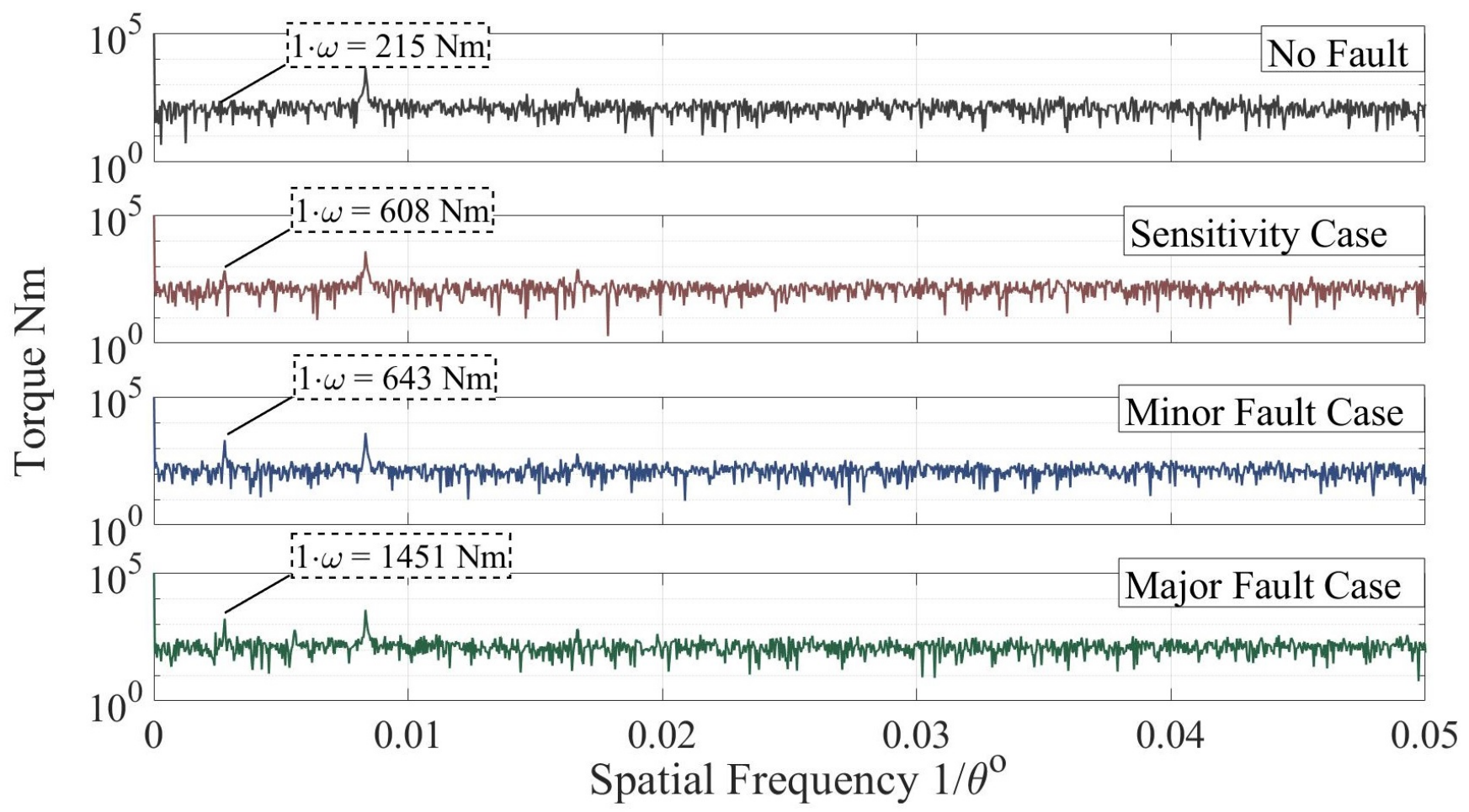

Figure 10. A sample of the spectra observed for the differing fault cases at a TI setting of $2 \%$.

\begin{tabular}{|c|c|c|c|c|c|c|c|c|c|c|c|}
\hline \begin{tabular}{|l} 
No \\
Fault \\
Case \\
TI $=2 \%$
\end{tabular} & $\begin{array}{l}\text { Sensitivity } \\
\text { Case } \\
\text { TI }=2 \%\end{array}$ & $\begin{array}{l}\text { Minor } \\
\text { Fault } \\
\text { Case } \\
\mathrm{TI}=2 \%\end{array}$ & $\begin{array}{l}\text { Major } \\
\text { Fault } \\
\text { Case } \\
\text { TI }=2 \%\end{array}$ & $\begin{array}{l}\text { No } \\
\text { Fault } \\
\text { Case } \\
\mathrm{TI}=1 \%\end{array}$ & $\begin{array}{l}\text { Sensitivity } \\
\text { Case } \\
\text { TI = } 1 \%\end{array}$ & $\begin{array}{l}\text { Minor } \\
\text { Fault } \\
\text { Case } \\
\mathrm{TI}=1 \%\end{array}$ & $\begin{array}{l}\text { Major } \\
\text { Fault } \\
\text { Case } \\
\text { TI }=1 \%\end{array}$ & $\begin{array}{l}\text { No } \\
\text { Fault } \\
\text { Case } \\
\text { TI }=0.5 \%\end{array}$ & $\begin{array}{l}\text { Sensitivity } \\
\text { Case } \\
\mathrm{TI}=0.5 \%\end{array}$ & $\begin{array}{l}\text { Minor } \\
\text { Fault } \\
\text { Case } \\
\text { TI }=0.5 \%\end{array}$ & $\begin{array}{l}\text { Major } \\
\text { Fault } \\
\text { Case } \\
\mathrm{TI}=0.5 \%\end{array}$ \\
\hline
\end{tabular}
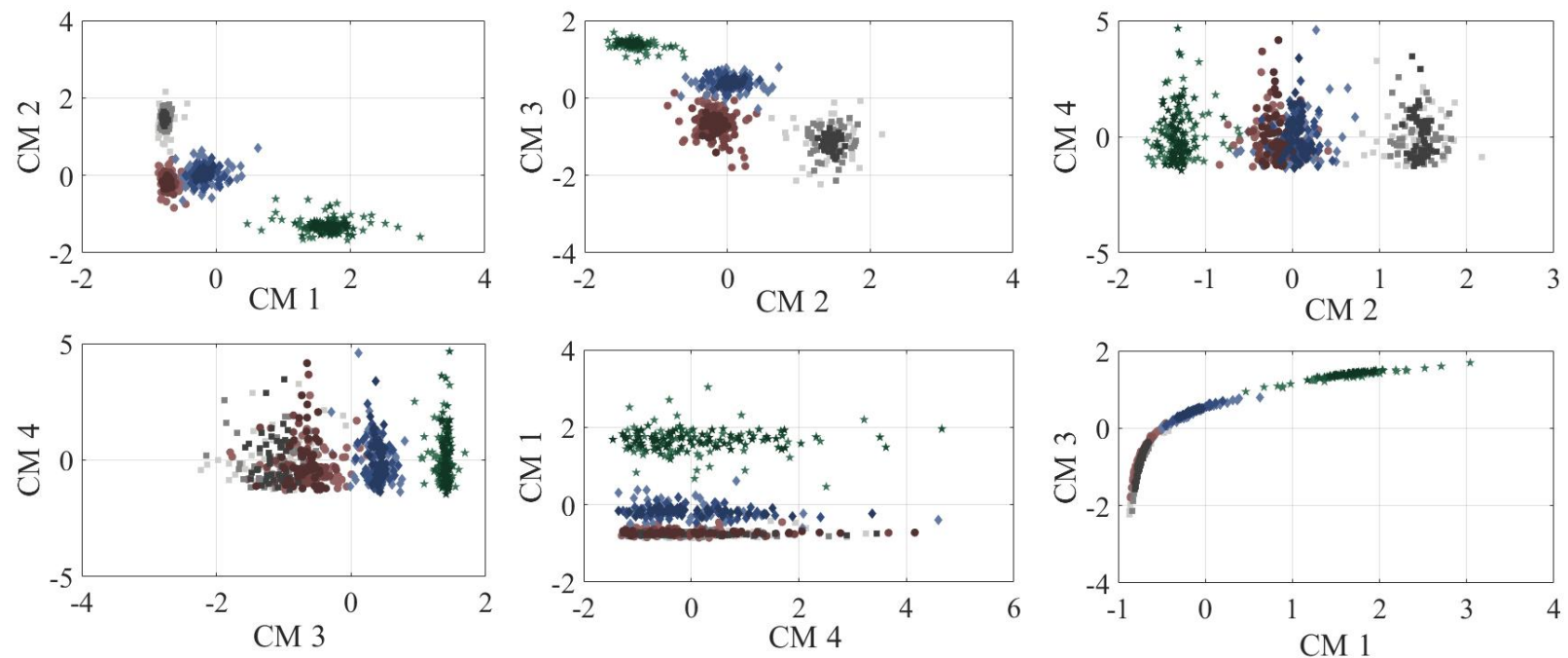

Figure 11. The results of the condition monitoring study. The plots show each of the CM metrics plotted against the other highlighting the structure of the 4-dimensional space created by the monitoring metrics. Each condition monitoring metric has been normalised by the subtracting the metric mean and dividing the data set by the metric standard deviation. 
fault case leading to correct classification under maximum a posteriori (MAP) classification.

Thus far in the development of the CM approach the use of CFD and the subsequent development of fault indicators has yielded a useful fault detection strategy. However, to fully test the developed approach further simulations were undertaken which included more a realistic fluid velocity model based on Kolmgrov's Theory of turbulence (Kolmogorov, 1941).

\section{Higher TurbulenCE INTENSity Results}

The testing of CFD models is often undertaken using laboratory scale devices. Under such conditions TI levels are limited. This is not the case in real life installations, where levels of $10 \%$ to $15 \%$ are more typical (Milne, Sharma, Flay, \& Bickerton, 2013). To further test the value of the CM method proposed it was necessary to consider the effect that the aforementioned TI characteristics would have on upon the CM approach.

\subsection{Extensions to the Condition Monitoring Study}

In order to test the use of the proposed monitoring approach under a more representative set of turbine operating conditions further simulations were undertaken. This set of simulations was undertaken utilising a more representative fluid velocity simulation process than that used in the simulations outlined in the sections above. All other aspects of the simulation, in terms of calculation process and model parametrisation, were kept the same. The specifics of the more advanced fluid velocity model can be found in the Appendix. Briefly, the model utilises a inverse-Fourier-Transfrom-Monte-Carlo approach to develop stochastic fluid velocity time series which approximately adhere to Kolmogorov's theory of turbulence (Kolmogorov, 1941). In essence this approach was used to generate time series which exhibited the $-5 / 3$ power spectra with a specific TI value and integral length scale. In this case the TI was set at $10 \%$ whilst the integral length scale was set to $40 \mathrm{~m}$.

\subsection{Simulation Results}

Ten extended simulations were undertaken, utilising the developed fluid velocity model, for each fault case. Figure 13 shows an instance of the simulations undertaken for each case; a sub axis has been included showing a 2 second section of the newly generated data. The simulations undertaken were 166 seconds in length with a time step of 0.005 s. Figure 13 shows the more chaotic nature of the time series generated with the new approach and shows that identification of the fault cases cannot be undertaken easily.

\subsection{Condition Monitoring Results}

As in Section 6 the spectra was calculated, via the FFT algorithm, for each simulation and fault case. The condition monitoring metrics were then calculated in accordance with the definitions outlined in Table 2. Figure 14 shows the spectra for the same simulation instances shown in Figure 13. The rotational $(1 \cdot \omega)$ and blade passing frequency $(3 \cdot \omega)$ associated with the simulated turbine have been highlighted on sub-axes. It can be seen that the characteristics identified in the CFD spectra outlined in Section 4 are far less discernible in the presence of the higher and more realistic turbulence spectra. To some degree this is expected as the more representative fluid velocity simulations involve creating a spectrum of random amplitude and phase angles which accordingly would disrupt the specific phasing relationship alluded to in Section 4.

Figure 15 shows the condition monitoring metrics calculated via the spectra of the simulated torque developed via the turbine rotor. The plots show each of the CM metrics plotted against the others highlighting the structure of the 4-dimension space created by the monitoring metrics. In this instance it can be easily observed that no such grouping of fault conditions can be seen based on the aforementioned monitoring metrics. This would suggest that the presence of the $10 \%$ turbulence, and its spectra consisting of random amplitudes and phases, has effectively mask the turbine operational frequencies.

Again to quantify the effectiveness of the CM metrics the datasets were used to train a naive Bayesian classifier set-up with equal prior probabilities for each fault case. The classifier was trained using a random sample of five of the ten data sets produced, with the correct label being given in each instance. Then the remaining five datasets was used to generate a prediction of the fault case via MAP inference. The bar chart in 16 shows the posterior probability given data input from each case. Figure 16 shows that only the 'No Fault' case is correctly diagnosed albeit with a low level of confidence. The Sensitivity case was classified as a 'No Fault Case', whereas both the 'Minor Fault' and the 'Major Fault cases were classified as the 'Sensitivity case'.

\section{Discussion}

The paper has, in three main sections, developed a condition monitoring strategy utilising CFD data and subsequently tested the approach using data generated via a parametric model. The approach was applied to data of relatively low turbulence intensity with a naive fluid velocity simulation process. The study was then extended by applying the approach to data generated with the aid of a more representative fluid velocity simulation approach.

Firstly, the process of utilising and extending the findings of CFD studies to generate CM insight given limited failure data relating to actual turbine deployments is discussed. The ap- 


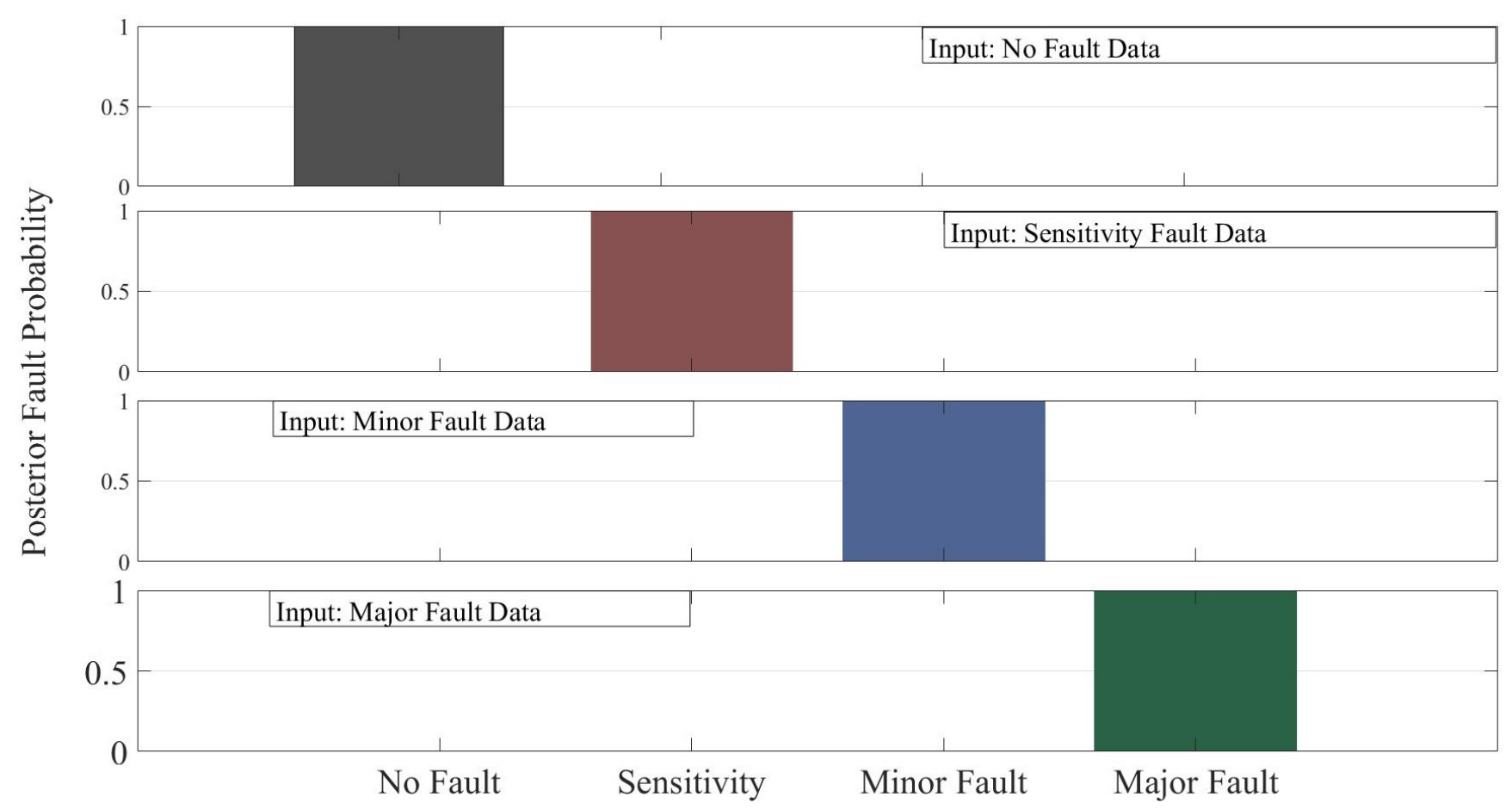

Figure 12. A series of bar charts showing the posterior probability of the fault case given the input data. This was calculated via a naive Bayesian classifier with equal prior probabilities for each fault case. The TI for the cases shown is $2 \%$

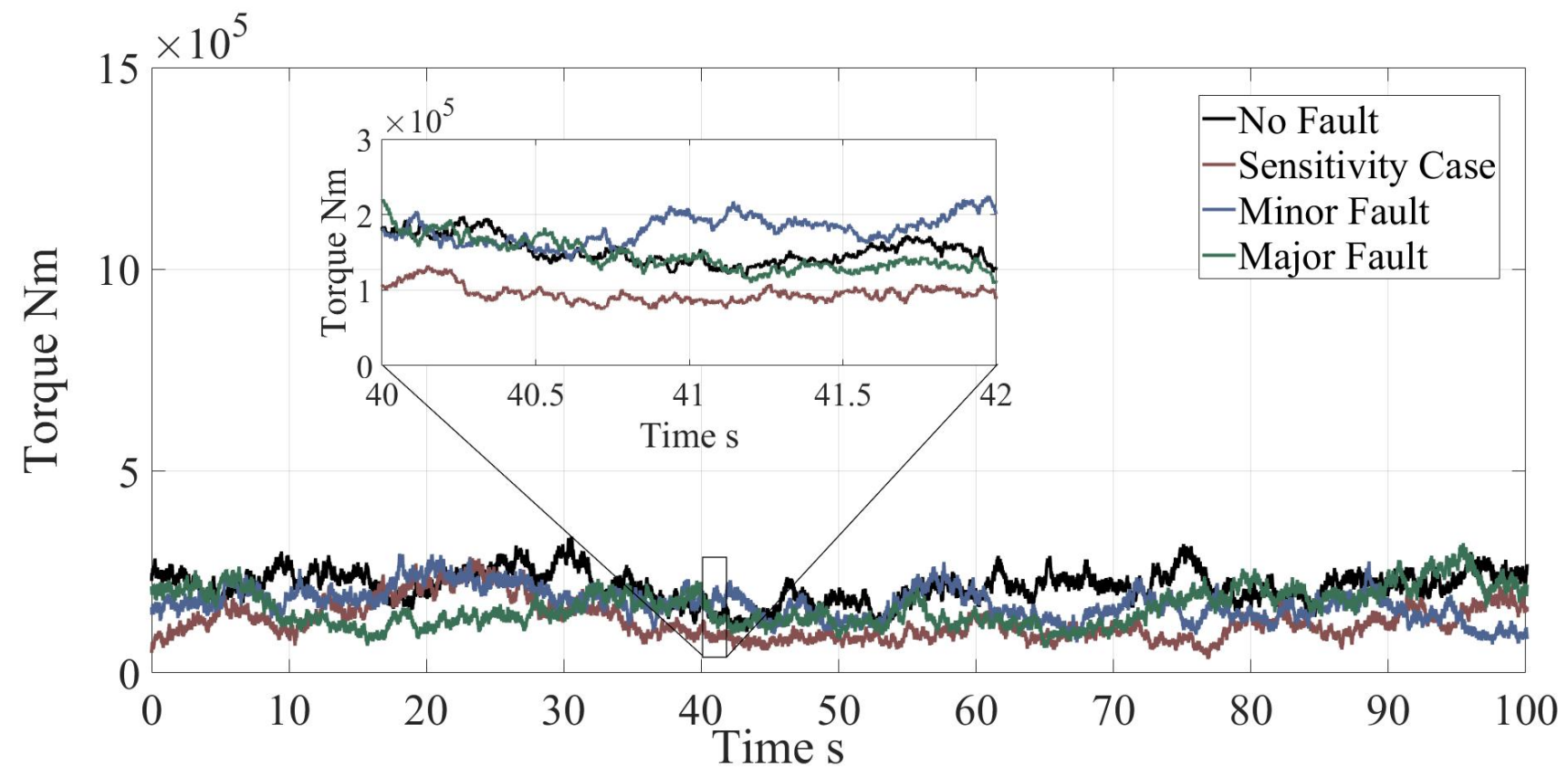

Figure 13. Time series of the model output, showing the simulated rotor torque under $10 \%$ TI in the on coming flow. 


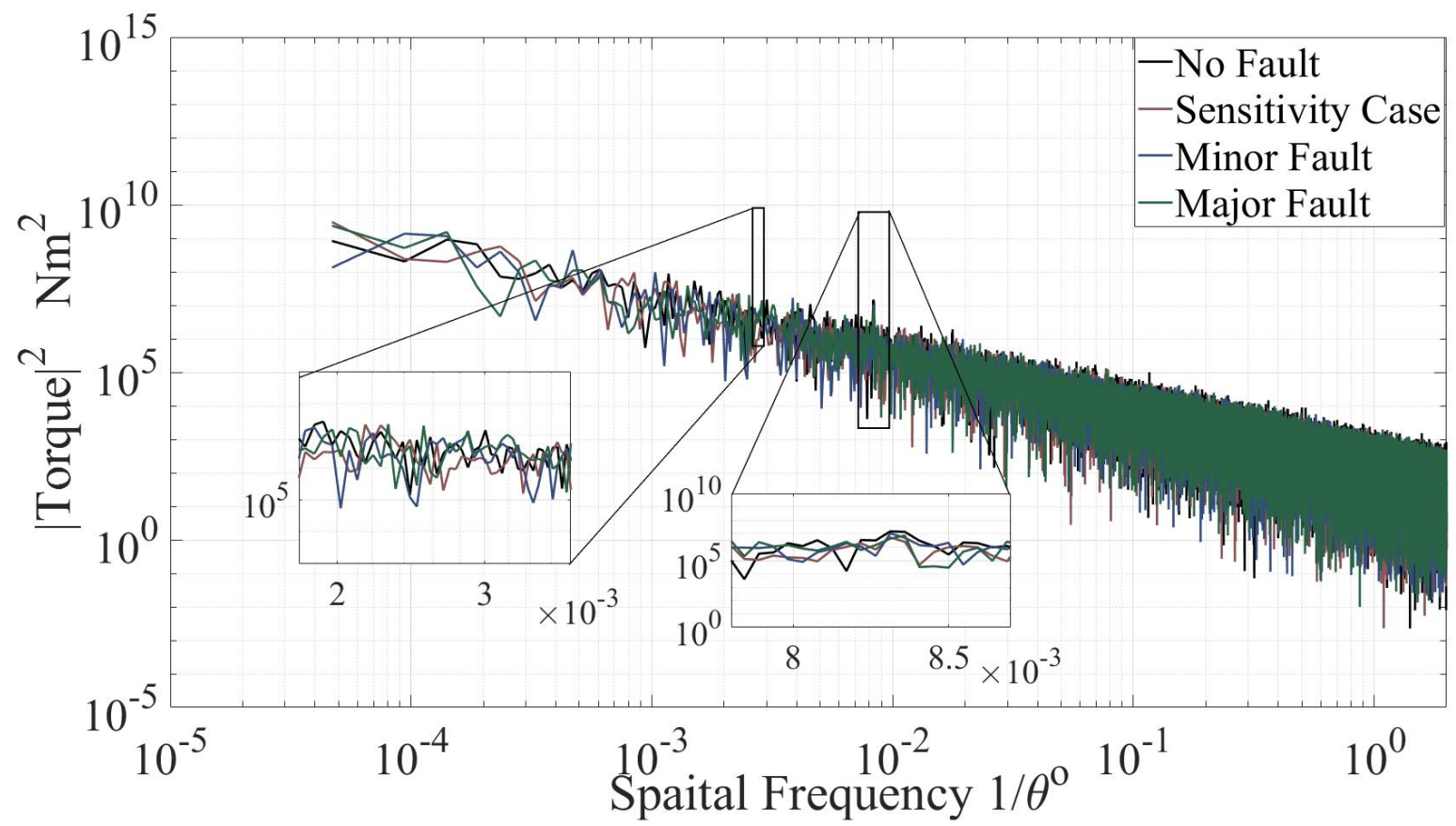

Figure 14. The spectra calculated for each fault case for the $10 \%$ TI simulations. The secondary axes show a more detailed view of the spectra at the rotational frequency and blade passing frequency of the turbine.

- No Fault $\bullet$ Sensitivity Fault $\star$ Minor Fault $\star$ Major Fault
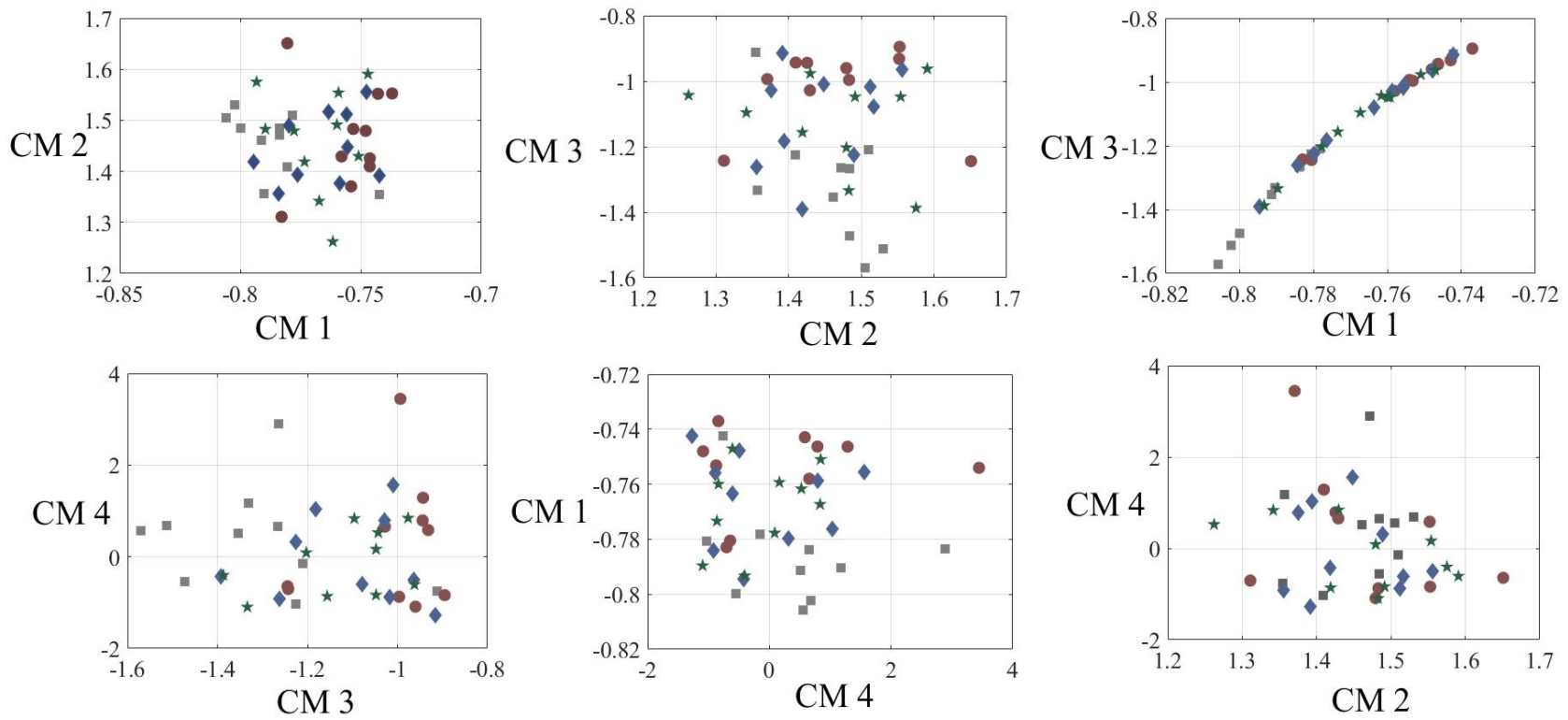

Figure 15 . The results of the condition monitoring study at $10 \% \mathrm{TI}$. 


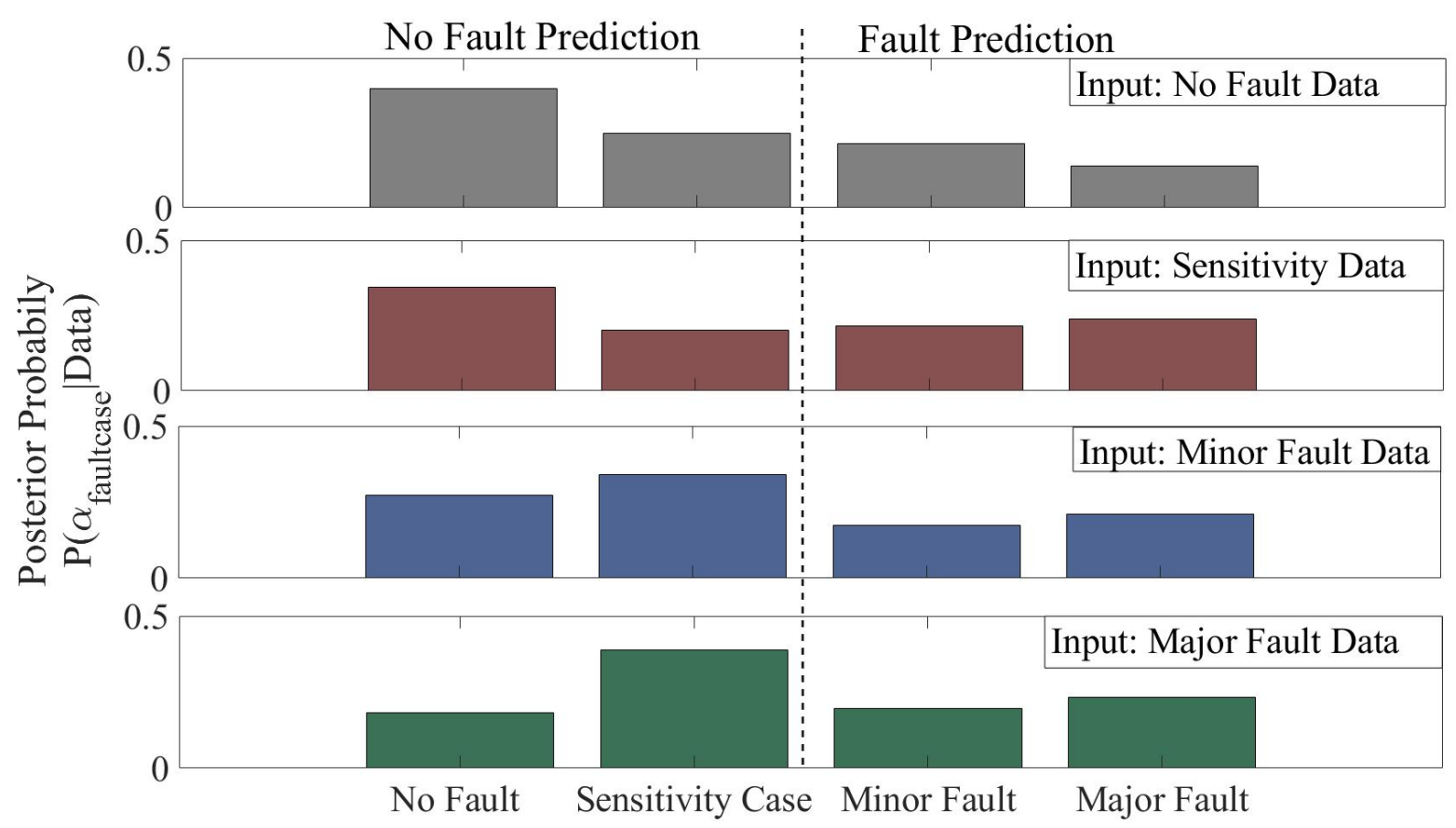

Figure 16. A series of bar charts showing the posterior probability of the fault case given the input data. This was calculated via a naive Bayesian classifier with equal prior probabilities for each fault case. The turbulence Intensity for the cases shown is $10 \%$

proach is promising as a means to generate understanding based on limited data and as a means of extending the usefulness of data generated via computationally expensive CFD studies. Whilst this is the case the authors acknowledge that rigorous validation of such an approach with experimental data must be undertaken before conclusive effectiveness of the approach can be heralded. This aspect of the work is currently being addressed in on-going research activities. The research involves defining a parameter set for a wide range of turbine operating conditions and comparing the statistical and spectral characteristics of the model output with experimental data.

Given the above discussion, the effectiveness of the CM approaches, developed and tested against the model output, will require further work before complete verification. The findings show that the high levels of turbulence intensity (approximately $10 \%$ ) significantly reduce the effectiveness of the presented CM approach. Whilst the results showed misdiagnosis for the $10 \%$ TI simulations, the CM approach is still under development and will required more rigorous data preprocessing. The overall methodology outlined has been successful in generating useful data sets relating to various rotor conditions and flow conditions and can be utilised subsequently during further developments of CM approaches for the HATT industry. Accordingly, the simulation data used for this set of testing has been made available to the researchers and can be accessed at the DOI specified in the Acknowledgement section.
The effectiveness of the approach under lower turbulence intensity conditions would suggest that the monitoring approach could be usefully applied during turbine start-up when turbulent kinetic energy in the on-coming flow lower, although further simulations are required to prove such an assertion. As such, and in the authors opinion, the work would suggest that whilst the approach may not be robust enough at this stage, further research into differing condition monitoring metrics and pre-processing approaches, coupled with larger data sets and more sensitive classification methods may yield a CM approach which may not require the installation of rotor based sensors.

\section{Conclusions}

The effectiveness of a CM approach, developed based on consideration of CFD data, under low TI simulations was shown. Under higher TIs in the on-coming flow the approach was not useful for fault detection. A methodology for extending CFD results to generate hypothetical failure data for HATTs has been created. The process was successfully applied to generated data relating to rotor imbalance faults. However, the model output must validated moving forward.

\section{FURTHER WORK}

Based on the results the authors are undertaking further research in four main respects. First, the authors are undertaking scale HATT testing to generate data sets under which the 
model proposed can be validated. Secondly, the authors are generating larger data sets to undertake more advanced CM research. The more advanced CM research forms the last two aspects of the on-going research activities. Specifically, condition monitoring metric formulation and pre-processing approaches are currently being researched, developed and tested. Lastly, these new methods are being tested in classification systems comprising of more sensitive Machine Learning techniques.

\section{ACKNOWLEDGEMENTS}

The authors acknowledge the financial support of EPSRC EP/N020782/1 under the Supergen Marine Grand Challenge programme. Information on the data underpinning the results presented here, including how to access them, can be found at Cardiff University data catalogue at

https://doi.org/10.17035/d.2018.0055962840

\section{REFERENCES}

Allmark, M. J. (2016). Condition monitoring and fault diagnosis of tidal stream turbines subjected to rotor imbalance faults (Unpublished doctoral dissertation). Cardiff University.

Caselitz, P., \& Giebhardt, J. (2005). Condition monitoring and fault prediction for marine current turbines. In International conference ocean energy; from innovation to industry (pp. 1-8).

Department of Energy \& Climate Change. (2013). DECC Electricity Generation Costs 2013 - GOV.UK (Tech. Rep.). London: Department of Energy \& Climate Change.

Frost, C., Morris, C. E., Mason-Jones, A., O’Doherty, D. M., \& O'Doherty, T. (2015, jun). The effect of tidal flow directionality on tidal turbine performance characteristics. Renewable Energy, 78, 609-620. doi: 10.1016/j.renene.2015.01.053

Godwin, J. I., \& Matthews, P. (2013). Classification and Detection of Wind Turbine Pitch Faults Through SCADA Data Analysis - PHM Society. IJPHM, 4(016).

Hasegawa, T., Ogata, J., Murakawa, M., Kobayashi, T., \& Ogawa, T. (2017). Adaptive training of vibration-based anomaly detector for wind turbine condition monitoring - PHM Society. IJPHM, 8(020).

Kolmogorov, A. (1941). The local structure of turbulence in incompressible viscous fluid for very large Reynolds numbers. Proceedings of the USSR Academy of Sciences, 30, 299-303.

Leahy, K., Hu, L. R., Konstantakopoulos, I. C., Spanos, C. J., Agogino, A. M., \& t.j. O'Sullivan, D. (2018). Diagnosing and Predicting Wind Turbine Faults from SCADA Data Using Support Vector Machines - PHM Society. IJPHM, 9(1).
Magagna, D., MacGillivray, A., Jeffrey, H., Hanmer, C., Raventos, A., \& Badcock-Broe, A. (2014). Wave and Tidal Energy Strategic Technology Agenda (Tech. Rep.). SI Ocean.

Mason-Jones, A. (2010). Performance assessment of a Horizontal Axis Tidal Turbine in a high velocity shear environment. (Unpublished doctoral dissertation). Cardiff University.

Mason-Jones, A., O'Doherty, D., Morris, C., O'Doherty, T., Byrne, C., Prickett, P., ... Poole, R. (2012, aug). Non-dimensional scaling of tidal stream turbines. Energy, 44(1), 820-829. doi: 10.1016/J.ENERGY.2012.05.010

Milne, I. A., Sharma, R. N., Flay, R. G. J., \& Bickerton, S. (2013, feb). Characteristics of the turbulence in the flow at a tidal stream power site. Philosophical transactions. Series A, Mathematical, physical, and engineering sciences, 371(1985), 20120196. doi: 10.1098/rsta.2012.0196

Mjit, M., Beaujean, P.-P. J., \& Vendittis, D. J. (2010, jan). Fault Detection and Diagnostics in an Ocean Turbine Using Vibration Analysis. In Volume 8: Dynamic systems and control, parts $a$ and $b$ (pp. 535-543). ASME. doi: 10.1115/IMECE2010-40963

Mjit Mustapha, Beaujean, P.-P., \& Vendittis, D. (2011). Comparison of Fault Detection Techniques for an Ocean Turbine - PHM Society. In Annual conference of the prognostics and health management society 2011. Montreal: PHM Society.

Sloan, J. C., Khoshgoftaar, T. M., Beaujean, P.-P., \& Driscoll, F. (2009, oct). OCEAN TURBINES A RELIABILITY ASSESSMENT. International Journal of Reliability, Quality and Safety Engineering, 16(05), 413-433. doi: 10.1142/S0218539309003472

Tatum, S., Allmark, M., Frost, C., O’Doherty, D., MasonJones, A., \& O'Doherty, T. (2016, sep). CFD modelling of a tidal stream turbine subjected to profiled flow and surface gravity waves. International Journal of Marine Energy, 15, 156-174. doi: 10.1016/J.IJOME.2016.04.003

Tatum, S., Frost, C., Allmark, M., O'Doherty, D., MasonJones, A., Prickett, P., ... O'Doherty, T. (2016, jun). Wavecurrent interaction effects on tidal stream turbine performance and loading characteristics. International Journal of Marine Energy, 14, 161-179. doi: 10.1016/J.IJOME.2015.09.002

Val, D. V., Chernin, L., \& Yurchenko, D. V. (2014). Reliability analysis of rotor blades of tidal stream turbines Reliability Engineering \& System Safety, 121, 26-33.

Waters, N., Beaujean, P.-P., \& Vendittis, D. (2013). Targeting Faulty Bearings for an Ocean Turbine Dynamometer - PHM Society. IJPHM, 4(021).

Weller, S., Thies, P., Gordelier, T., \& Johanning, L. (2015, nov). Reducing Reliability Uncertainties 
for Marine Renewable Energy. Journal of Marine Science and Engineering, 3(4), 1349-1361. doi: $10.3390 / \mathrm{jmse} 3041349$

Wolfram, J. (2006, jan). On Assessing the Reliability and Availability of Marine Energy Converters: The Problems of a New Technology. Proceedings of the Institution of Mechanical Engineers, Part O: Journal of Risk and Reliability, 220(1), 55-68. doi: 10.1243/1748006XJRR7

Zhang, X.-P., \& Zeng, P. (2013, apr). Marine Energy Technology [Sanning the Issue]. Proceedings of the IEEE, 101(4), 862-865. doi: 10.1109/JPROC.2013.2244735

\section{BIOGRAPHIES}

Dr Matthew Allmark Educated at Cardiff University obtaining a BEng in Mechanical Engineering (2011) and a PhD on the condition monitoroing of tidal stream turbines. $\mathrm{He}$ is a post doctoral research within the Cardiff Marine Energy group currently undertaking research considering the dynamic loading of tidal stream turbines and how this impacts the monitoring and maintenance of such devices.

Paul Prickett Educated at Cardiff University obtaining a BEng degree in Mechanical Engineering (1979). He is a senior lecturer in Cardiff School of Engineering and is also a codirector of the IPMM centre (established in 1998). He has directed the work of sixteen researchers and has supervised seven PhD students and two EngD students. He has published over 70 papers on the topic of machine and process condition monitoring. He at presented at numerous conferences and acts as a referee for journals and funding bodies in his field of research. He is a chartered engineer and a member of the Institution of Mechanical Engineers.

Dr Roger I Grosvenor Educated at Cardiff University obtaining a BEng Mechanical Engineering degree (1978), a MEng via fluid dynamics research (1981) and a PhD on the topic of in-process measurement of machined components (1994). He is currently a reader in systems engineering at Cardiff University and has been employed as a lecturer there since 1984 . He has published 90 journal and conference papers, mainly on the topic of machine and process condition monitoring. He is co-director of the Intelligent Process monitoring and management (IPMM) centre. He is a chartered engineer and a member of the Institute of Measurement and Control.
Dr Carwyn Frost Dr Carwyn Frost is a Research Fellow in the Marine Research Group within Queens University Belfast (QUB). Educated at Cardiff University with a BEng in Mechanical Engineering (2012) and a PhD on the numerical modelling of tidal stream turbines (2017). Most recently Dr Frost is engaged in post-doctoral research in experimental tidal testing of a $1.5 \mathrm{~m}$ diameter tidal turbine in the QUB tidal test site in Strangford Narrows, Northern Ireland and the CNRINSEAN towing tank facility in Rome, Italy.

\section{APPEndix - Resource Simulation}

The simulations outlined in this paper use the simplification that the turbine would be subjected to plug flow (non-profile flow) conditions. This simplification was necessary as this was the approximate flow conditions observed during the flume testing campaign used to gather data for the model parametrisation. The plug flow assumption leads to a convenient representation of the flow conditions hitting the turbine rotor. The flow is represented by:

$$
U_{x}(t)=\bar{U}_{x}+u_{x}^{\prime}(t)
$$

where $U_{x}(t)$ is the fluid velocity at time $\mathrm{t}$ decomposed into a stationary mean fluid velocity $\bar{U}_{x}$ and a fluctuating component $u_{x}^{\prime}(t)$, which is time varying with the $\mathrm{x}$ direction perpendicular to the turbine rotor plane. A natural model for representing the fluid flow given by the above is to model the fluid velocity fluctuations as a stationary process with given power spectral density characteristics. Furthermore utilising Kolomogrovs theory of turbulence the amplitude of the power spectrum should be proportional to $f^{-5 / 3}$ as $f \rightarrow \infty$. The Von Karman spectrum, as utilised by previous investigators (Val et al., 2014) for reliability simulations adheres to the above condition and can be written in the non-dimensional form:

$$
\frac{f S_{u}(f)}{\sigma_{u}^{2}}=\frac{\frac{4 f L}{U_{x}}}{\left[1+70.79\left(\frac{f L}{\bar{U}_{x}}\right)^{2}\right]^{\frac{5}{6}}}
$$

where $S u(f)$ is the spectral density function for the process, $L$ is the length scale, $\sigma_{u}$ is the standard deviation of the process $u_{x}(t)$. More information on the development of fluid velocity simulations can be found in (Allmark, 2016) 